Homology, Homotopy and Applications, vol.18(2), 2016, pp.283-301

\title{
MOTIVIC AND DERIVED MOTIVIC HIRZEBRUCH CLASSES
}

\author{
JEAN-PAUL BRASSELET, JÖRG SCHÜRMANN AND SHOJI YOKURA
}

(communicated by Jonathan Rosenberg)

\begin{abstract}
In this paper we give a formula for the Hirzebruch $\chi_{y}$-genus $\chi_{y}(X)$ and similarly for the motivic Hirzebruch class $T_{y *}(X)$ for possibly singular varieties $X$, using the Vandermonde matrix. Motivated by the notion of secondary Euler characteristic and higher Euler characteristic, we consider a similar notion for the motivic Hirzebruch class, which we call a derived motivic Hirzebruch class.
\end{abstract}

\section{Introduction}

First we will recall that the Euler-Poincaré characteristic is a kind of "generalization" or "broad extension" of the counting of a finite set, where the counting of a finite set $X$ is the so-called cardinality, i.e.,

$$
c(X):=|X|=\text { the number of the elements in the set } X .
$$

Certainly the counting $c$ for finite sets satisfies the following basic properties:

1. $A \cong A^{\prime}$ (bijection or equipotent) $\Longrightarrow c(A)=c\left(A^{\prime}\right)$,

2. $c(A)=c(A \backslash B)+c(B)$ for $B \subset A$ (this is called "scissor formula" or "motivic"),

3. $c(A \times B)=c(A) \cdot c(B)$,

4. $c(p t)=1$. (Here $p t$ denotes one point.)

Now, if we consider the following "topological counting" $c$ on the category of some "nice" topological spaces such that $c(X) \in \mathbb{Z}$ and it satisfies the following four properties:

- $X \cong X^{\prime}$ (homeomorphism $=\mathcal{T} \mathcal{O P}$-isomorphism) $\Longrightarrow c(X)=c\left(X^{\prime}\right)$,

- $c(X)=c(X \backslash Y)+c(Y)$ for $Y \subset X$,

- $c(X \times Y)=c(X) \cdot c(Y)$,

- $c(p t)=1$,

The second author was supported in part by the SFB 878 groups, geometry and actions. The third author has been supported in part by JSPS KAKENHI Grant Numbers 24340007, 24540085, $16 \mathrm{H} 03936$.

Received December 17, 2015, revised March 3, 2016; published on November 2, 2016.

2010 Mathematics Subject Classification: 14C17, 14C40, 14F25, 14F45, 14Q15, 32S35.

Key words and phrases: higher Euler characteristic, arithmetic genus, signature, Hirzebruch genus, homology, Chern class, Todd class, $L$-class, motivic Hirzebruch class.

Article available at http://dx.doi.org/10.4310/HHA.2016.v18.n2.a16

Copyright (C) 2016, International Press. Permission to copy for private use granted. 
then one can show that if such a $c$ exists, then we must have that

$$
c\left(\mathbb{R}^{1}\right)=-1, \text { hence } c\left(\mathbb{R}^{n}\right)=(-1)^{n} .
$$

Hence, if $X$ is a finite $C W$-complex with $\sigma_{n}(X)$ denoting the number of open $n$-cells, then

$$
c(X)=\sum_{n}(-1)^{n} \sigma_{n}(X)=\chi(X)
$$

is the Euler-Poincaré characteristic of $X$. Namely, the topological counting $c$ is uniquely determined and it is the compactly supported Euler-Poincaré characteristic.

Remark 1.1. 1. Such a counting is not defined for all topological spaces, as one can see for example that such a $c$ is not well-defined on the discrete space $\mathbb{Z}$ of integers. Such a counting is defined on "nice" spaces such as finite $C W$ complexes. Here we do not bother ourselves to specify what we mean by "nice" (cf. Peters' TATA Lecture Notes [25]).

2. It would be safe to say that the reason why the Euler-Poincaré characteristic (which is the very fundamental, basic but still important topological invariant in topology, geometry and physics) is defined by the alternating sum of the numbers of vertices, edges, faces, and so on,

$$
\chi(X):=V-E+F-\cdots
$$

comes from our usual simple "counting".

Now, let us consider such a counting on the category $\mathcal{V}$ of algebraic varieties:

- $X \cong X^{\prime}(\mathcal{V}$-isomorphism $) \Longrightarrow c(X)=c\left(X^{\prime}\right)$,

- $c(X)=c(X \backslash Y)+c(Y)$ for a closed subvariety $Y \subset X$,

- $c(X \times Y)=c(X) \cdot c(Y)$,

- $c(p t)=1$.

If such an "algebraic" counting $c$ exists, then it follows from the decomposition of the $n$-dimensional complex projective space

$$
\mathbb{P}^{n}=\mathbb{C}^{0} \sqcup \mathbb{C}^{1} \sqcup \cdots \sqcup \mathbb{C}^{n-1} \sqcup \mathbb{C}^{n},
$$

that we must have

$$
c\left(\mathbb{P}^{n}\right)=1-y+y^{2}-y^{3}+\cdots+(-y)^{n},
$$

where $y:=-c\left(\mathbb{C}^{1}\right) \in \mathbb{Z}$. In fact, it follows from Deligne's mixed Hodge structure that the following Hodge--Deligne polynomial

$$
\chi_{u, v}(X):=\sum_{i, p, q \geqslant 0}(-1)^{i}(-1)^{p+q} \operatorname{dim}_{\mathbb{C}}\left(G r_{F}^{p} G r_{p+q}^{W} H_{c}^{i}(X, \mathbb{C})\right) u^{p} v^{q}
$$

satisfies the above four properties, namely any Hodge-Deligne polynomial $\chi_{u, v}$ with $u v=-y$ is such a $c$. The Hirzebruch $\chi_{y}$ characteristic is nothing but $\chi_{y,-1}$ and the most important and interesting ones are the following:

- $y=-1: \quad \chi_{-1}=\chi$, the topological Euler-Poincaré characteristic, 
- $y=0: \quad \chi_{0}=\chi^{a}$, the arithmetic genus (for a compact nonsingular variety),

- $y=1: \quad \chi_{1}=\sigma$, the signature (for a compact nonsingular variety).

Remark 1.2. Here we note that $\chi_{0}=\chi^{a}$ also holds for $X$ compact with at most Du Bois singularities (by $[\mathbf{3}]$ ) and $\chi_{1}=\sigma$ for $X$ a projective rational homology manifold (by $[22, \S 3.6]$ ).

It turns out (see $[\mathbf{3}, \mathbf{3 4}]$ ) that the Hodge-Deligne polynomial $\chi_{u, v}: K_{0}(\mathcal{V}) \rightarrow \mathbb{Z}[u, v]$ can be extended as a class version only when $u=y, v=-1$, just like HirzebruchRiemann-Roch was extended by Grothendieck as a natural transformation from the covariant functor of coherent sheaves to the rational homology theory, which is called Grothendieck-Riemann-Roch. Here $K_{0}(\mathcal{V})$ is the Grothendieck group of complex algebraic varieties with respect to the scissor relation. Namely, only the Hirzebruch $\chi_{y}$ characteristic

$$
\chi_{y}: K_{0}(\mathcal{V}) \rightarrow \mathbb{Z}[y]
$$

can be extended as a class version

$$
T_{y *}: K_{0}(\mathcal{V} / X) \rightarrow H_{*}(X) \otimes \mathbb{Q}[y] .
$$

This is called the motivic Hirzebruch class. Here $K_{0}(\mathcal{V} / X)$ is the relative Grothendieck group of complex algebraic varieties as recalled in $\S 3$ and $H_{*}(X)$ is the Borel-Moore homology group.

In this paper we give some formulas for the motivic Hirzebruch class and its "derived version", which we call "derived motivic Hirzebruch class", motivated by higher Euler characteristic generalizing the secondary characteristic (see [26] and cf. $[8])$.

\section{Hirzebruch $\chi_{y}$-genus and Hirzebruch class $T_{y}$}

First we recall the definition of the Hirzebruch $\chi_{y}$-genus. Let $X$ be a smooth complex projective variety. The $\chi_{y}$-genus of $X$ is defined by

$$
\chi_{y}(X):=\sum_{p \geqslant 0} \chi\left(X, \Lambda^{p} T^{*} X\right) y^{p}=\sum_{p \geqslant 0}\left(\sum_{i \geqslant 0}(-1)^{i} \operatorname{dim}_{\mathbb{C}} H^{i}\left(X, \Lambda^{p} T^{*} X\right)\right) y^{p} .
$$

Thus the $\chi_{y}$-genus is the generating function of the Euler-Poincaré characteristic $\chi\left(X, \Lambda^{p} T^{*} X\right)$ of the $p$-th exterior power $\Lambda^{p} T^{*} X$ of the cotangent bundle $T^{*} X$, which shall be simply denoted by $\chi^{p}(X)$ :

$$
\chi_{y}(X)=\sum_{p \geqslant 0} \chi^{p}(X) y^{p} .
$$

Since $\Lambda^{p} T^{*} X=0$ for $p>\operatorname{dim}_{\mathbb{C}} X, \chi_{y}(X)$ is a polynomial of at most degree $\operatorname{dim}_{\mathbb{C}} X$.

More generally, for $E$ a holomorphic vector bundle over $X$, the Hirzebruch $\chi_{y}$-genus of $E$ is defined by 
$\chi_{y}(X, E):=\sum_{p \geqslant 0} \chi\left(X, E \otimes \Lambda^{p} T^{*} X\right) y^{p}=\sum_{p \geqslant 0}\left(\sum_{i \geqslant 0}(-1)^{i} \operatorname{dim}_{\mathbb{C}} H^{i}\left(X, E \otimes \Lambda^{p} T^{*} X\right)\right) y^{p}$.

Then we have

$$
\chi_{y}(X, E)=\int_{X} T_{y}(T X) \cdot \operatorname{ch}_{(1+y)}(E) \cap[X] \quad \in \mathbb{Q}[y]
$$

$$
\text { with } \quad \operatorname{ch}_{(1+y)}(E):=\sum_{j=1}^{\operatorname{rank} E} e^{\beta_{j}(1+y)} \text { and } T_{y}(T X):=\prod_{i=1}^{\operatorname{dim} X} Q_{y}\left(\alpha_{i}\right) .
$$

Here $\beta_{j}$ are the Chern roots of $E, \alpha_{i}$ are the Chern roots of the tangent bundle $T X$, and $Q_{y}(\alpha)$ is the normalized power series

$$
Q_{y}(\alpha):=\frac{\alpha(1+y)}{1-e^{-\alpha(1+y)}}-\alpha y \quad \in \mathbb{Q}[y][[\alpha]] .
$$

Note that this power series $Q_{y}(\alpha)$ specializes to

$$
Q_{y}(\alpha)= \begin{cases}1+\alpha & \text { for } y=-1 \\ \frac{\alpha}{1-e^{-\alpha}} & \text { for } y=0 \\ \frac{\alpha}{\tanh \alpha} & \text { for } y=1\end{cases}
$$

Therefore the modified Todd class $T_{y}(T X)$ unifies the following important characteristic cohomology classes of $T X$ :

$$
T_{y}(T X)= \begin{cases}c(T X) & \text { the total Chern class for } y=-1 \\ t d(T X) & \text { the total Todd class for } y=0 \\ L(T X) & \text { the total Thom-Hirzebruch L-class for } y=1 .\end{cases}
$$

We call the modified Todd class $T_{y}(T X)$ the Hirzebruch class of $X$.

The coefficient of the power $y^{p}$ of the Hirzebruch class $T_{y}(E)$ shall be denoted by $T^{p}(E)$ (cf. Hirzebruch's book $\left.[\mathbf{1 0}]\right)$ :

$$
T_{y}(E)=\sum_{i=0}^{\operatorname{rank} E} T^{p}(E) y^{p} .
$$

Here we emphasize that each $T^{p}(E)$ is a polynomial of Chern classes or Pontryagin classes without the variable $y$ not involved at all.

The Hirzebruch $\chi_{y}$-genus is by the generalized Hirzebruch-Riemann-Roch formula given by

$$
\chi_{y}(X)=\int_{X} T_{y}(T X) \cap[X] .
$$

Hence for a compact nonsingular variety $X$ of dimension $n$

$$
\chi_{y}(X)=\sum_{i=0}^{n}\left(\int_{X} T^{p}(T X) \cap[X]\right) y^{p} .
$$


So we note that

$$
\chi^{p}(X)=\int_{X} T^{p}(T X) \cap[X]
$$

Since we eventually deal with homology classes, we define

$$
T_{*}^{p}(X):=T^{p}(T X) \cap[X], \quad T_{y *}(X):=\sum_{p=0}^{n} T_{*}^{p}(X) y^{p} .
$$

For the distinguished three values $-1,0,1$ of $y$, by the definition we have the following:

- $c(E)=T_{-1}(E)=T^{0}(E)-T^{1}(E)+T^{2}(E)-\cdots+(-1)^{n} T^{\mathrm{rank} E}(E)$,

- $t d(E)=T_{0}(E)=T^{0}(E)$,

- $L(E)=T_{1}(E)=T^{0}(E)+T^{1}(E)+T^{2}(E)+\cdots+T^{\mathrm{rank} E}(E)$,

- $\chi(X)=\chi_{-1}(X)=\chi^{0}(X)-\chi^{1}(X)+\chi^{2}(X)-\cdots+(-1)^{n} \chi^{n}(X)$,

- $\chi^{a}(X)=\chi_{0}(T X)=\chi^{0}(X)$,

- $\sigma(X)=\chi_{1}(T X)=\chi^{0}(X)+\chi^{1}(X)+\chi^{2}(X)+\cdots+\chi^{n}(X)$.

Remark 2.1. 1. Each individual coefficient $T^{p}(E)$ of the Hirzebruch class $T_{y}(E)$ may be computed from the very definition of it, but as the above formulas for these three distinguished values suggest, certain summations of all these coefficients make more sense than each individual $T^{p}(E)$ geometrically or topologically. It is the same for the Hirzebruch $\chi_{y}$-genus $\chi_{y}(X)$.

2. It would be worthwhile to observe that

$$
\begin{aligned}
& \frac{L(E)+c(E)}{2}=T^{0}(E)+T^{2}(E)+T^{4}(E)+\cdots: \quad \text { the "even part", } \\
& \frac{L(E)-c(E)}{2}=T^{1}(E)+T^{3}(E)+T^{5}(E)+\cdots: \quad \text { the "odd part". }
\end{aligned}
$$

3. It follows from $[\mathbf{1 0}]$ that for a compact nonsingular variety $X$

$$
\chi^{p}(X)=(-1)^{n} \chi^{n-p}(X) .
$$

It is also known (e.g., see $[\mathbf{1 4}]$ and cf. $[\mathbf{1 3}]$ ) that $\chi^{0}, \chi^{1}, \cdots, \chi^{\left[\frac{n}{2}\right]}$ are linearly independent, which means that for any compact nonsingular variety $X$ of dimension $n$

$$
r_{0} \chi^{0}(X)+r_{1} \chi^{1}(X)+\cdots+r_{\left[\frac{n}{2}\right]} \chi^{\left[\frac{n}{2}\right]}(X)=0
$$

implies that $r_{0}=r_{1}=\cdots=r_{\left[\frac{n}{2}\right]}=0$. As a corollary of this, we can also say that $T_{*}^{0}, T_{*}^{1}, \cdots, T_{*}^{\left[\frac{n}{2}\right]}$ are linearly independent, otherwise the linear dependence of $T_{*}^{0}, T_{*}^{1}, \cdots, T_{*}^{\left[\frac{n}{2}\right]}$ implies the linear dependence of $\chi^{0}, \chi^{1}, \cdots, \chi^{\left[\frac{n}{2}\right]}$, which contradicts the above linear independence.

4. The above "duality formula" $\chi^{p}(X)=(-1)^{n} \chi^{n-p}(X)$ implies the following "inversion formula": for a compact nonsingular variety $X$

$$
\chi_{y}(X)=(-y)^{n} \chi_{\frac{1}{y}}(X), \quad \text { i.e., } \quad \chi_{\frac{1}{y}}(X)=\left(-\frac{1}{y}\right)^{n} \chi_{y}(X) .
$$




\section{Motivic Hirzebruch classes $T_{y *}$}

The Hirzebruch $\chi_{y}$-genus was extended to the case of singular varieties, using Deligne's mixed Hodge structures, i.e.,

$$
\begin{aligned}
\chi_{y}(X) & :=\sum_{i, p \geqslant 0}(-1)^{i} \operatorname{dim}_{\mathbb{C}} G r_{\mathcal{F}}^{p}\left(H_{c}^{i}(X, \mathbb{C})\right)(-y)^{p} \\
& =\sum_{p \geqslant 0}\left(\sum_{i \geqslant 0}(-1)^{i+p} \operatorname{dim}_{\mathbb{C}} G r_{\mathcal{F}}^{p}\left(H_{c}^{i}(X, \mathbb{C})\right)\right) y^{p} .
\end{aligned}
$$

Here $\mathcal{F}$ is the Hodge filtration in the mixed Hodge structure of $H_{c}^{i}(X, \mathbb{C})$. Thus for a possibly singular variety $X$, the coefficient $\chi^{p}(X)$ of the above Hirzebruch $\chi_{y^{-}}$genus $\chi_{y}(X)$ is

$$
\chi^{p}(X)=\sum_{i \geqslant 0}(-1)^{i+p} \operatorname{dim}_{\mathbb{C}} G r_{\mathcal{F}}^{p}\left(H_{c}^{i}(X, \mathbb{C})\right) .
$$

Here we remark that the degree of the above integral polynomial $\chi_{y}(X)$ of a possibly singular variety is also at most the dimension of $X$ like in the smooth case (cf. [3, Corollary 3.1(1)]).

The three distinguished characteristic classes have been extended to the case of possibly singular varieties as natural transformations from certain covariant functors to the homology functor. This formulation is analogous to the interpretation that the classical theory of characteristic classes of vector bundles is a natural transformation from the contravariant monoid functor $\mathcal{V}$ ect or the Grothendieck $K$-theory of real or complex vector bundles to the contravariant cohomology theory. The three theories of characteristic classes of singular varieties are the following:

1. MacPherson's Chern class transformation [19]:

$$
c_{*}: F(X) \rightarrow H_{*}(X),
$$

where $F$ is the covariant functor assigning to $X$ the abelian group $F(X)$ of constructible functions on $X$. Here we remark that Brasselet and Schwartz [5] (see also [1]) showed that MacPherson's Chern class $c_{*}\left(\mathbb{1}_{X}\right)$ corresponds to the Schwartz class $c^{S}(X) \in H_{X}^{*}(M)=H^{*}(M, M \backslash X)$ (see [31, 32]) by Alexander duality for $X$ embedded in the smooth complex manifold $M$. That is why the total homology class $c_{*}(X):=c_{*}\left(\mathbb{1}_{X}\right)$ is called the Chern-SchwartzMacPherson class of $X$.

2. Baum-Fulton-MacPherson's Todd class or Riemann-Roch [2]:

$$
t d_{*}: G_{0}(X) \rightarrow H_{*}(X) \otimes \mathbb{Q},
$$

where $G_{0}$ is the covariant functor assigning to $X$ the Grothendieck group $G_{0}(X)$ of coherent sheaves on $X$.

3. Goresky-MacPherson's homology $L$-class [9], which is extended as a natural transformation by Cappell and Shaneson [6] (also see [33]):

$$
L_{*}: \Omega(X) \rightarrow H_{*}(X) \otimes \mathbb{Q},
$$

where $\Omega$ is the covariant functor assigning to $X$ the cobordism group $\Omega(X)$ of self-dual constructible sheaf complexes on $X$. 
In our previous paper $[\mathbf{3}]$ (see also $[\mathbf{4}, \mathbf{3 0}, \mathbf{2 9}, \mathbf{3 4}]$ ) we introduced the motivic Hirzebruch class

$$
T_{y_{*}}: K_{0}(\mathcal{V} / X) \rightarrow H_{*}(X) \otimes \mathbb{Q}[y]
$$

where $K_{0}(\mathcal{V} / X)$ is the relative Grothendieck group of the category $\mathcal{V}$ of complex algebraic varieties, i.e., the free abelian group generated by the isomorphism classes $[V \stackrel{h}{\rightarrow} X]$ of morphism $h \in h_{\mathcal{V}}(V, X)$ modulo the relations:

- $\left[V_{1} \stackrel{h_{1}}{\longrightarrow} X\right]+\left[V_{2} \stackrel{h_{2}}{\longrightarrow} X\right]=\left[V_{1} \sqcup V_{2} \stackrel{h_{1}+h_{2}}{\longrightarrow} X\right]$, with $\sqcup$ the disjoint union, and

- $[V \stackrel{h}{\rightarrow} X]=\left[V \backslash W \stackrel{h_{V \backslash W}}{\longrightarrow} X\right]+\left[W \stackrel{h_{W}}{\longrightarrow} X\right]$ for $W \subset V$ a closed subvariety of $V$.

$T_{y_{*}}: K_{0}(\mathcal{V} / X) \rightarrow H_{*}(X) \otimes \mathbb{Q}[y]$ is the unique natural transformation satisfying the normalization condition that if $X$ is nonsingular, then

$$
T_{y_{*}}\left[X \stackrel{\operatorname{id}_{X}}{\longrightarrow} X\right]=T_{y}(T X) \cap[X] .
$$

Here $T_{y}(T X)$ is the above Hirzebruch class.

Our $T_{y_{*}}: K_{0}(\mathcal{V} / X) \rightarrow H_{*}(X) \otimes \mathbb{Q}[y]$ "unifies" the above three characteristic classes $c_{*}, t d_{*}, L_{*}$ in the sense that we have the following commutative diagrams:
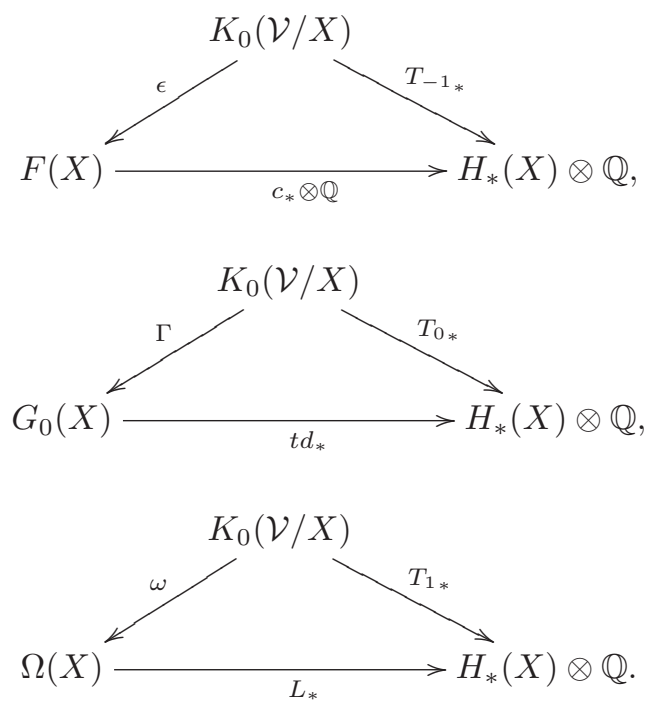

This "unification" could be considered as a positive answer to the following remark which is stated at the very end of MacPherson's survey article [20] (which is a paper version of his survey talk about characteristic classes of singular varieties at Brazilian Math. Colloquium in 1973): "It remains to be seen whether there is a unified theory of characteristic classes of singular varieties like the classical one outlined above."1

\footnotetext{
${ }^{1}$ At that time Goresky-MacPherson's homology L-class was not available yet and it was defined only after the theory of Intersection Homology was invented by Mark Goresky and Robert MacPherson in 1980 .
} 


\section{Naive explicit formulae for $\chi_{y}(X)$ and $T_{y *}(X)$}

\subsection{A natural question}

Whenever we have given talks about the above motivic Hirzebruch class and $\chi_{y^{-}}$ genus, commenting or emphasizing that our $T_{y_{*}}: K_{0}(\mathcal{V} / X) \rightarrow H_{*}(X) \otimes \mathbb{Q}[y]$ unifies the above three characteristic classes $c_{*}, t d_{*}, L_{*}$, the Hirzebruch class $T_{y}(E)$ specialize to the well-known three distinguished ones: Chern class, Todd class and $L$-class, thus the Hirzebruch $\chi_{y}$-genus specializes to Euler-Poincaré characteristic, the arithmetic genus and the signature for $y=-1,0,1$, respectively, we always have been asked the following question:

Question 4.1. How about other values $\chi_{y}$ and $T_{y *}$ for $y \neq-1,0,1$, say, at other integers?

A motivation of the present work is trying to answer this very reasonable and natural question. Although we have been unable to give a complete answer, the one we give in this paper would be a reasonable one at the moment, considering the fact that (as far as we know) there is no literature available of concrete or explicit formula for $\chi_{y}(X)$ for an even smooth variety $X$ and for general integers $y$. The idea of our formula is quite simple, because $\chi_{y}(X)$ and $T_{y *}(X)$ are both polynomials of $y$ of finite degree. Then such a polynomial can be completely described using the special values at $\operatorname{dim} X+1$ points, using the Interpolation Formula or the Vandermonde matrix.

\subsection{Interpolation polynomial and Vandermonde}

Here we recall some basic things for the sake of completeness.

Let $f: \mathbb{R} \rightarrow \mathbb{R}$ be a given function and let $\left\{a_{i}\right\}(0 \leqq i \leqq n)$ be mutually distinct points, i.e., $a_{i} \neq a_{j}(i \neq j)$. An interpolation polynomial for the function $f$ is determined by the following Lagrange interpolation polynomial:

$$
p(x)=\sum_{i=0}^{n}\left(\prod_{0 \leqq j \leqq n, j \neq i} \frac{x-a_{j}}{a_{i}-a_{j}}\right) f\left(a_{i}\right) .
$$

Expressing the above interpolation polynomial in the form of

$$
p(x)=p_{0}+p_{1} x+p_{2} x^{2}+\cdots+p_{n} x^{n}
$$

can be done directly by using the Vandermonde matrix. Indeed, we have the following linear equations:

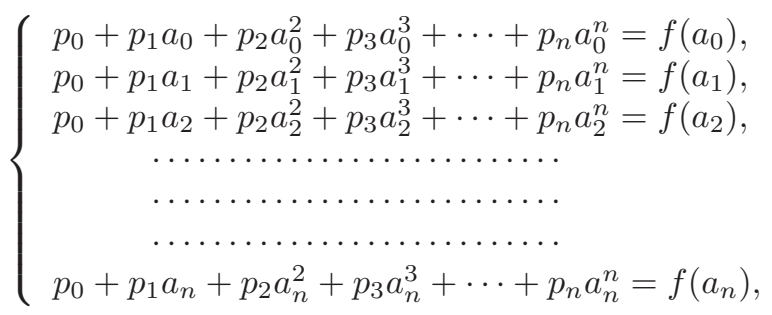


i.e.,

$$
\left(\begin{array}{ccccc}
1 & a_{0} & a_{0}^{2} & a_{0}^{3} \ldots \ldots & a_{0}^{n} \\
1 & a_{1} & a_{1}^{2} & a_{1}^{3} \ldots \cdots & a_{1}^{n} \\
1 & a_{2} & a_{2}^{2} & a_{2}^{3} \ldots \cdots & a_{2}^{n} \\
\vdots & & \ddots & & \vdots \\
1 & a_{n} & a_{n}^{2} & a_{n}^{3} \ldots \ldots & a_{n}^{n}
\end{array}\right)\left(\begin{array}{c}
p_{0} \\
p_{1} \\
p_{2} \\
\vdots \\
p_{n}
\end{array}\right)=\left(\begin{array}{c}
f\left(a_{0}\right) \\
f\left(a_{1}\right) \\
f\left(a_{2}\right) \\
\vdots \\
f\left(a_{n}\right)
\end{array}\right)
$$

Let $V\left(a_{0}, a_{1}, a_{2}, \cdots, a_{n}\right)$ be the above Vandermonde matrix. The determinant of this Vandermonde is

$$
\operatorname{det}\left(V\left(a_{0}, a_{1}, a_{2}, \cdots, a_{n}\right)\right)=\prod_{0 \leqq i<j \leqq n}\left(a_{j}-a_{i}\right),
$$

which is $\neq 0$ since $a_{i} \neq a_{j}(i \neq j)$. Therefore the coefficients $p_{0}, p_{1}, p_{2}, \cdots, p_{n}$ can be determined by the following equation, by computing the inverse of the Vandermonde $V\left(a_{0}, a_{1}, a_{2}, \cdots, a_{n}\right)$ :

$$
\left(\begin{array}{c}
p_{0} \\
p_{1} \\
p_{2} \\
\vdots \\
p_{n}
\end{array}\right)=\left(\begin{array}{ccccc}
1 & a_{0} & a_{0}^{2} & a_{0}^{3} \cdots \cdots & a_{0}^{n} \\
1 & a_{1} & a_{1}^{2} & a_{1}^{3} \cdots \cdots & a_{1}^{n} \\
1 & a_{2} & a_{2}^{2} & a_{2}^{3} \cdots \cdots & a_{2}^{n} \\
\vdots & & \ddots & & \vdots \\
1 & a_{n} & a_{n}^{2} & a_{n}^{3} \cdots \cdots & a_{n}^{n}
\end{array}\right)^{-1}\left(\begin{array}{c}
f\left(a_{0}\right) \\
f\left(a_{1}\right) \\
f\left(a_{2}\right) \\
\vdots \\
f\left(a_{n}\right)
\end{array}\right)
$$

Remark 4.2. We note that the above Lagrange interpolation formula and the method of using the inverse of the Vandermonde matrix can be applied to any function from $\mathbb{R}(\mathbb{C}$, resp.) to any vector space $\mathcal{V E C}$ over $\mathbb{R}(\mathbb{C}$, resp.).

\subsection{Explicit computations}

In this section we give an explicit formula for Hirzebruch's $\chi_{y}$-genus $\chi_{y}(X)$ and the Hirzebruch class $T_{y}(X)$ for a compact nonsingular variety $X$ of dimension $n$. Here we recall that:

- $T_{y *}(X)=T_{*}^{0}(X)+T_{*}^{1}(X) y+T_{*}^{2}(X) y^{2}+\cdots+T_{*}^{n}(X) y^{n} \in H_{*}(X) \otimes \mathbb{Q}[y]$,

- $\chi_{y}(X)=\chi^{0}(X)+\chi^{1}(X) y+\chi^{2}(X) y^{2}+\cdots+\chi^{n}(X) y^{n} \in \mathbb{Q}[y]$.

We just deal with $\chi_{y}(X)$ since it is exactly the same for $T_{y *}(X)$.

Example 4.3. $n=1: \chi_{y}(X)=\chi^{0}(X)+\chi^{1}(X) y$. Since we have

$$
\chi(X)=\chi^{0}(X)-\chi^{1}(X), \quad \chi^{a}(X)=\chi^{0}(X), \quad \sigma(X)=\chi^{0}(X)+\chi^{1}(X),
$$

we get that $\chi^{1}(X)=\frac{\sigma(X)-\chi(X)}{2}$. Here we note that the signature $\sigma(X)=0$ by definition, since it is defined to be zero if the real dimension $\operatorname{dim}_{\mathbb{R}} X \not \equiv 0 \bmod 4$. Hence we have

$$
\chi_{y}(X)=\chi^{a}(X)-\frac{\chi(X)}{2} y
$$

Here we also note that

$$
\chi^{a}(X)=\frac{\chi(X)}{2}
$$


Example 4.4. $n=2: \chi_{y}(X)=\chi^{0}(X)+\chi^{1}(X) y+\chi^{2}(X) y^{2}$. Since we have

$$
\begin{gathered}
\chi(X)=\chi^{0}(X)-\chi^{1}(X)+\chi^{2}(X), \quad \chi^{a}(X)=\chi^{0}(X), \\
\sigma(X)=\chi^{0}(X)+\chi^{1}(X)+\chi^{2}(X),
\end{gathered}
$$

we get that $\chi^{1}(X)=\frac{\sigma(X)-\chi(X)}{2}, \chi^{2}(X)=\frac{\chi(X)+\sigma(X)-2 \chi^{a}(X)}{2}$. Hence we have

$$
\chi_{y}(X)=\chi^{a}(X)+\frac{\sigma(X)-\chi(X)}{2} y+\frac{\chi(X)+\sigma(X)-2 \chi^{a}(X)}{2} y^{2} .
$$

Since the Hirzebruch $\chi_{y}$-genus is multiplicative and the Hirzebruch homology class $T_{*}$ is also multiplicative, i.e., respectively

$$
\chi_{y}(X \times Y)=\chi_{y}(X) \cdot \chi_{y}(Y) \quad \text { and } \quad T_{y *}(X \times Y)=T_{y *}(X) \times T_{y *}(Y),
$$

we obtain the following formulas:

Theorem 4.5. Let $C_{i}(1 \leqq i \leqq s)$ be a compact nonsingular curve and $S_{j}(1 \leqq j \leqq t)$ be a compact nonsingular surface. Then we have the following formulae:

$$
\begin{aligned}
& \chi_{y}\left(C_{1} \times C_{2} \times \cdots \times C_{s} \times S_{1} \times S_{2} \times \cdots \times S_{t}\right) \\
& =\prod_{i=1}^{s}\left(\chi^{a}\left(C_{i}\right)-\frac{\chi\left(C_{i}\right)}{2} y\right) \cdot \prod_{j=1}^{t}\left(\chi^{a}\left(S_{j}\right)+\frac{\sigma\left(S_{j}\right)-\chi\left(S_{j}\right)}{2} y+\frac{\sigma\left(S_{j}\right)+\chi\left(S_{j}\right)-2 \chi^{a}\left(S_{j}\right)}{2} y^{2}\right), \\
& T_{y *}\left(C_{1} \times C_{2} \times \cdots \times C_{s} \times S_{1} \times S_{2} \times \cdots \times S_{t}\right) \\
& \quad=\prod_{i=1}^{s}\left(T d_{*}\left(C_{i}\right)+\frac{L_{*}\left(C_{i}\right)-c_{*}\left(C_{i}\right)}{2} y\right) \times \\
& \quad \prod_{j=1}^{t}\left(T d_{*}\left(S_{j}\right)+\frac{L_{*}\left(S_{j}\right)-c_{*}\left(S_{j}\right)}{2} y+\frac{L_{*}\left(S_{j}\right)+c_{*}\left(S_{j}\right)-2 T d_{*}\left(S_{j}\right)}{2} y^{2}\right) .
\end{aligned}
$$

Here $T d_{*}, L_{*}$ and $c_{*}$ are, respectively, the Todd homology class, the L-homology class and the Chern homology class, i.e., the Poincaré dual of the corresponding characteristic class.

In particular, the constant coefficients and the top degree coefficients of them are, respectively, the following:

$$
\begin{aligned}
& \chi^{0}\left(C_{1} \times \cdots \times C_{s} \times S_{1} \times \cdots \times S_{t}\right)=\prod_{i=1}^{s} \chi^{a}\left(C_{i}\right) \cdot \prod_{j=1}^{t} \chi^{a}\left(S_{j}\right), \\
& \chi^{s+2 t}\left(C_{1} \times \cdots \times C_{s} \times S_{1} \times \cdots \times S_{t}\right)=\prod_{i=1}^{s} \frac{-\chi\left(C_{i}\right)}{2} \cdot \prod_{j=1}^{t} \frac{\sigma\left(S_{j}\right)+\chi\left(S_{j}\right)-2 \chi^{a}\left(S_{j}\right)}{2}, \\
& T_{y *}^{0}\left(C_{1} \times \cdots \times C_{s} \times S_{1} \times \cdots \times S_{t}\right)=\prod_{i=1}^{s} T d_{*}\left(C_{i}\right) \times \prod_{j=1}^{t} T d_{*}\left(S_{j}\right)
\end{aligned}
$$




$$
\begin{aligned}
T_{y *}^{s+2 t}\left(C_{1} \times \cdots \times C_{s} \times S_{1} \times \cdots \times S_{t}\right)=\prod_{i=1}^{s} \frac{L_{*}\left(C_{i}\right)-c_{*}\left(C_{i}\right)}{2} \times \\
\prod_{j=1}^{t} \frac{L_{*}\left(S_{j}\right)+c_{*}\left(S_{j}\right)-2 T d_{*}\left(S_{j}\right)}{2} .
\end{aligned}
$$

Here, as to the $T_{y *}^{0}$ and $T_{y *}^{s+2 t}$, the product $\prod_{i=1}^{s} T d_{*}\left(C_{i}\right)$ means the cross product and the same for the other products.

Remark 4.6. As far as the degree zero part and the top degree part are concerned, for the product of any varieties we can get the following formulae:

$\begin{array}{ll}\text { 1. } \chi^{0}\left(X_{1} \times \cdots \times X_{n}\right)=\prod_{i=1}^{n} \chi^{a}\left(X_{i}\right), \quad \chi^{\sum_{i} \operatorname{dim} X_{i}}\left(X_{1} \times \cdots \times X_{n}\right)=\prod_{i=1}^{n} \chi^{\operatorname{dim} X_{i}}\left(X_{i}\right), \\ \text { 2. } T_{y *}^{0}\left(X_{1} \times \cdots \times X_{n}\right)=\prod_{i=1}^{n} T d_{*}\left(X_{i}\right), & T_{y *}^{\sum_{i} \operatorname{dim} X_{i}}\left(X_{1} \times \cdots \times X_{n}\right)=\prod_{i=1}^{n} T_{y *}^{\operatorname{dim} X_{i}}\left(X_{i}\right) .\end{array}$

In (2) the product $\prod_{i=1}^{n}$ is the cross product as in the above corollary.

In the case when $n \geqq 3$, we need $n+1 \geqq 4$ points, thus the three points $-1,0,1$ are not enough.

Theorem 4.7. Let $X$ be a compact nonsingular variety of dimension $n$. Let $a_{0}=0$, $a_{1}=1, a_{2}=-1, a_{3}, \cdots, a_{n}$ be mutually distinct numbers. Then we have

$$
\chi_{y}(X)=\chi^{0}(X)+\chi^{1}(X) y+\chi^{2}(X) y^{2}+\cdots+\chi^{n}(X) y^{n},
$$

where

$$
\left(\begin{array}{c}
\chi^{0}(X) \\
\chi^{1}(X) \\
\chi^{2}(X) \\
\chi^{3}(X) \\
\vdots \\
\chi^{n-1}(X) \\
\chi^{n}(X)
\end{array}\right)=\left(\begin{array}{ccccc}
1 & 0 & 0 & 0 \cdots \cdots & 0 \\
1 & 1 & 1 & 1 \cdots \cdots & 1 \\
1 & -1 & (-1)^{2} & (-1)^{3} \cdots \cdots & (-1)^{n} \\
1 & a_{3} & a_{3}^{2} & a_{3}^{3} \cdots \cdots & a_{3}^{n} \\
\vdots & & \ddots & & \vdots \\
1 & a_{n-1} & a_{n-1}^{2} & a_{n-1}^{3} \ldots \ldots & a_{n-1}^{n} \\
1 & a_{n} & a_{n}^{2} & a_{n}^{3} \cdots \cdots & a_{n}^{n}
\end{array}\right)^{-1}\left(\begin{array}{c}
\chi^{a}(X) \\
\sigma(X) \\
\chi(X) \\
\chi_{a_{3}}(X) \\
\vdots \\
\chi_{a_{n-1}}(X) \\
\chi_{a_{n}}(X)
\end{array}\right)
$$

Example 4.8. Let $n=3$ and $a_{3}=2$. (Here we note that the signature $\sigma(X)=0$ since $\operatorname{dim}_{\mathbb{R}} X=6 \not \equiv 0 \bmod 4$.)

$$
\begin{aligned}
\chi_{y}(X)=\chi^{a}(X)+\frac{-2 \chi(X)-}{3 \chi^{a}(X)-\chi_{2}(X)} & 6 \\
& +\frac{\chi(X)-2 \chi^{a}(X)}{2} y^{2}+\frac{\chi(X)+3 \chi^{a}(X)+\chi_{2}(X)}{6} y^{3} .
\end{aligned}
$$

Remark 4.9. Using the inversion formula in Remark 2.1(4), for example, if $n=2 k$, it suffices to consider the Vandermonde $V_{n+1}\left(0,1,-1,2,3, \cdots, k, 2^{-1}, 3^{-1} \cdots, k^{-1}\right)$ and the special values $\chi_{0}(X)=\chi^{a}(X), \chi_{1}(X)=\sigma(X), \chi_{-1}(X)=\chi(X), \chi_{2}(X), \chi_{3}(X)$, 
- and $\chi_{k}(X)$.

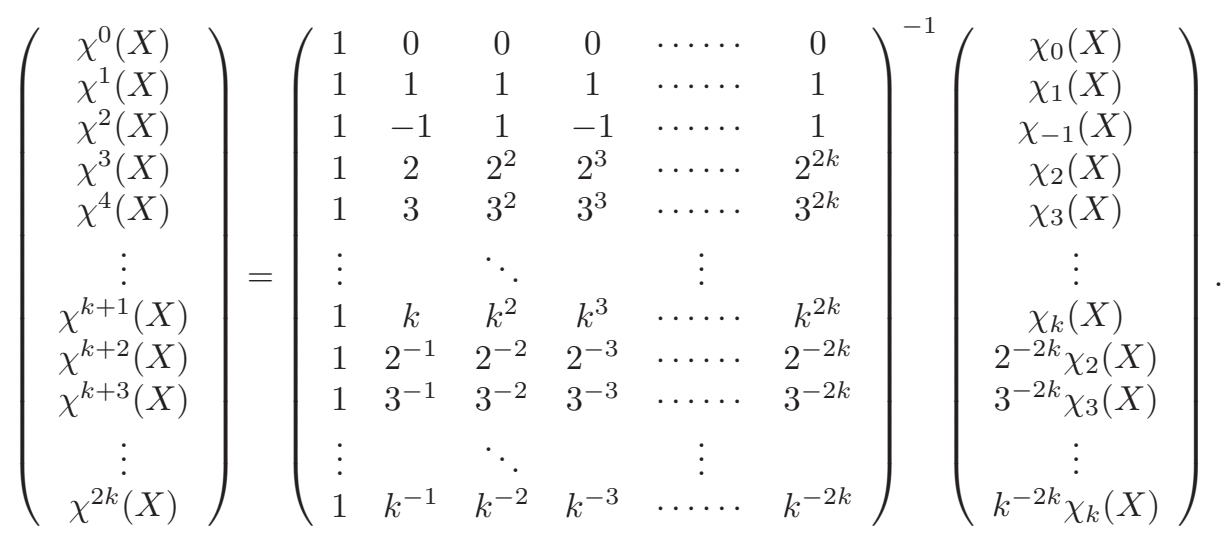

Question 4.10. Let $i$ be any integer greater than 1. Can one express $\chi_{i}(X)$ and $\chi_{-i}(X)$ in terms of some other known invariants such as the Euler-Poincaré characteristic $\chi(X)$, the arithmetic genus $\chi^{a}(X)$, the signature $\sigma(X)$ and so on?

Theorem 4.7 also holds for the motivic Hirzebruch class $T_{y *}(X)=T_{y *}\left(\left[X \stackrel{\mathrm{id}_{X}}{\longrightarrow} X\right]\right)$ for any possibly singular variety $X$ of dimension $n$ :

Theorem 4.11. Let $X$ be a possibly singular variety of dimension $n$. Let $a_{0}=0$, $a_{1}=1, a_{2}=-1, a_{3}, \cdots, a_{n}$ be mutually distinct numbers. Then we have the following formula:

$$
T_{y *}(X)=T_{*}^{0}(X)+T_{*}^{1}(X) y+T_{*}^{2}(X) y^{2}+\cdots+T_{*}^{n}(X) y^{n},
$$

where

$$
\left(\begin{array}{c}
T_{*}^{0}(X) \\
T_{*}^{1}(X) \\
T_{*}^{2}(X) \\
T_{*}^{3}(X) \\
\vdots \\
T_{*}^{n-1}(X) \\
T_{*}^{n}(X)
\end{array}\right)=\left(\begin{array}{ccccc}
1 & 0 & 0 & 0 \cdots \cdots & 0 \\
1 & 1 & 1 & 1 \cdots \cdots & 1 \\
1 & -1 & (-1)^{2} & (-1)^{3} \cdots \cdots & (-1)^{n} \\
1 & a_{3} & a_{3}^{2} & a_{3}^{3} \cdots \cdots & a_{3}^{n} \\
\vdots & & \ddots & & \vdots \\
1 & a_{n-1} & a_{n-1}^{2} & a_{n-1}^{3} \ldots \cdots & a_{n-1}^{n} \\
1 & a_{n} & a_{n}^{2} & a_{n}^{3} \cdots \cdots & a_{n}^{n}
\end{array}\right)^{-1}\left(\begin{array}{c}
T_{0 *}(X) \\
T_{1 *}(X) \\
c_{*}(X) \otimes \mathbb{Q} \\
T_{a_{3} *}(X) \\
\vdots \\
T_{a_{n-1} *}(X) \\
T_{a_{n} *}(X)
\end{array}\right)
$$

We get the following corollary from $[\mathbf{3}, \mathbf{2 1}]$ :

Corollary 4.12. Let the situation be as in Theorem 4.11.

1. If $X$ is a toric variety, then $T_{0 *}(X)$ can be replaced by Baum-Fulton-MacPherson's Todd class $t d_{*}(X)$.

2. If $X$ is a simplicial projective toric variety, then $T_{0 *}(X)$ can be replaced by Baum-Fulton-MacPherson's Todd class $t d_{*}(X)$ and furthermore $T_{1 *}(X)$ can be replaced by Cappell-Shaneson's homology L-class $L_{*}(X)$. 


\section{Derived Hirzebruch $\chi_{y}$-genus and derived motivic Hirzebruch class}

As stated above, the first motivation is trying to get a general formula of the Hirzebruch $\chi_{y}$-genus as well as the motivic Hirzebruch class $T_{y *}(X)$, using the special values of them at $y=-1,0,1$ and some other points, the secondary motivation of the present paper is as follows:

The Euler-Poincaré characteristic $\chi(X)$ is the alternating sum of the Betti numbers, i.e., $\chi(X)=\sum_{i}(-1)^{i} \operatorname{dim} H_{i}(X ; \mathbb{R})$. If we use the Poincaré polynomial $P_{X}(t):=$ $\sum_{i} \operatorname{dim} H_{i}(X ; \mathbb{R}) t^{i}$, then we have

$$
\chi(X)=P_{X}(-1) .
$$

Namely, the Euler-Poincaré characteristic $\chi(X)$ is the constant term $a_{0}=P_{X}(-1)$ of the Taylor expansion of the Poincaré polynomial $P_{X}(t)$ at $t=-1$ :

$$
P_{X}(t)=a_{0}+a_{1}(t+1)+a_{2}(t+1)^{2}+\cdots+a_{k}(t+1)^{k}+\cdots a_{n}(t+1)^{n},
$$

where $n$ is the degree of the Poincaré polynomial. More precisely $a_{k}=\frac{P_{X}^{(k)}(-1)}{k !}$. The coefficient $a_{1}$ is called the secondary Euler characteristic, denoted by $\chi^{(1)}(X)$ and the other coefficients $a_{k}$ shall be called the $k$-th higher Euler characteristic and denoted by $\chi^{(k)}(X)$ (cf. Ramachandran's recent paper [26] and [8]). We shall understand the Chern-Schwartz-MacPherson class $c_{*}(X)$ to be a homology class version of the Euler-Poincaré characteristic, then a very natural question is the following:

Question 5.1. Is there a homology class version of the $k$-th higher Euler characteristic $\chi^{(k)}(X)$ ?

To be able to answer this question, if we could have a certain reasonable "Poincaré polynomial" for a compact variety $X$

$$
\mathfrak{P}_{X}(t) \in H_{*}(X ; \mathbb{R})[t],
$$

such that

1. $\mathfrak{P}_{X}(t)=c_{*}^{S M}(X)+\mathfrak{a}_{1}(t+1)+\mathfrak{a}_{2}(t+1)^{2}+\cdots+\mathfrak{a}_{k}(t+1)^{k}+\cdots \mathfrak{a}_{n}(t+1)^{n}$,

2. $\int_{X} \mathfrak{P}_{X}(t)=P_{X}(t)$, i.e., $\int_{X} c_{*}^{S M}(X)=\chi(X)$ and $\int_{X} \mathfrak{a}_{k}=a_{k}$,

then $\mathfrak{a}_{k}$ would be such a homology class version of the $k$-th higher Euler characteristic $\chi^{(k)}(X)$.

At the moment we do not know if there would be such a "Poincaré polynomial" $\mathfrak{P}_{X}(t)$, although we have MacPherson's Chern class transformation $c_{*}: F(X) \rightarrow$ $H_{*}(X)$. However, in the case of the Hirzebruch $\chi_{y}$-genus $\chi_{y}(X)$, which is a polynomial such that the special value of $\chi_{y}(X)$ at $y=-1$, i.e., $\chi_{-1}(X)$ is the Euler-Poincaré characteristic, we have the Taylor expansion of $\chi_{y}(X)$ at $y=-1$

$$
\chi_{y}(X)=\chi_{-1}(X)+a_{1}(X)(y+1)+a_{2}(X)(y+1)^{2}+a_{3}(X)(y+1)^{3}+\cdots .
$$

It turns out that many people (e.g., see $[\mathbf{1 7}, \mathbf{2 4}, \mathbf{2 8}, \mathbf{1 2}, \mathbf{1 5}, \mathbf{1 6}]$, etc.) have studied the first few coefficients $a_{1}(X), a_{2}(X), a_{3}(X), a_{4}(X)$. Thus, motivated by this fact, we also consider such coefficients for the motivic Hirzebruch classes, which we call derived motivic Hirzebruch classes. 
First we consider the following general situation. Given a polynomial of degree $n$ :

$$
f(y)=b_{0}+b_{1} y+b_{2} y^{2}+\cdots+b_{p} y^{p}+\cdots+b_{n} y^{n},
$$

we consider the Taylor expansion of $f(y)$ at $y=\alpha$ :

$$
f(y)=a_{0}+a_{1}(y-\alpha)+a_{2}(y-\alpha)^{2}+\cdots+a_{p}(y-\alpha)^{p}+\cdots+a_{n}(y-\alpha)^{n},
$$

where each coefficient $a_{p}$ can be expressed as follows

$$
a_{p}=\frac{f^{(p)}(\alpha)}{p !} .
$$

We can see the following relations between $a_{i}$ 's and $b_{j}$ 's:

$$
a_{p}=\sum_{k=p}^{n}\left(\begin{array}{l}
k \\
p
\end{array}\right) b_{k} \alpha^{k-p} \quad \text { or } \quad b_{p}=\sum_{k=p}^{n}\left(\begin{array}{l}
k \\
p
\end{array}\right) a_{k}(-\alpha)^{k-p} .
$$

Expressing them in the matrix forms, we have

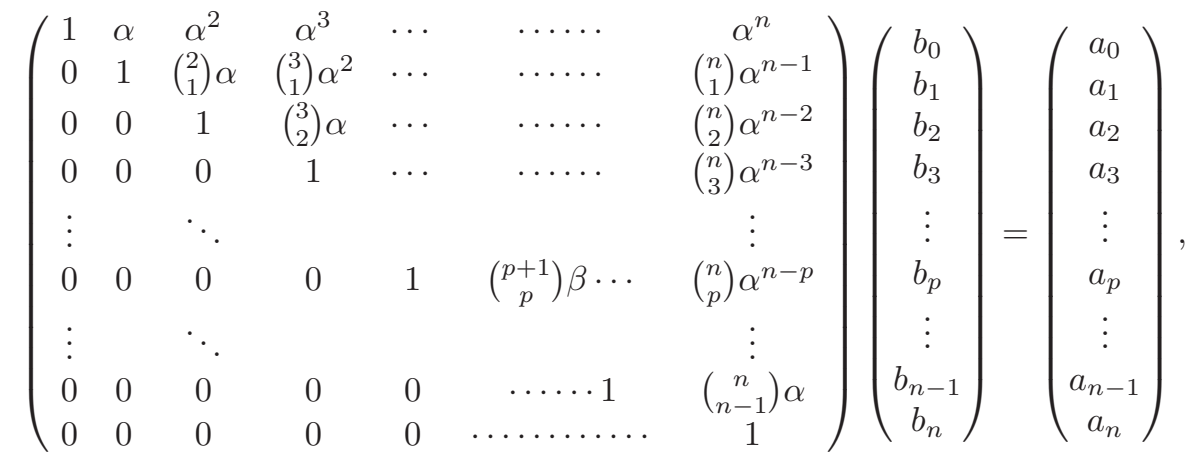

$$
\begin{aligned}
& \left(\begin{array}{ccccccc}
1 & -\alpha & \alpha^{2} & -\alpha^{3} & \cdots & \cdots \cdots & (-\alpha)^{n} \\
0 & 1 & -\left(\begin{array}{c}
2 \\
1
\end{array}\right) \alpha & \left(\begin{array}{l}
3 \\
1
\end{array}\right) \alpha^{2} & \cdots & \cdots \cdots & \left(\begin{array}{l}
n \\
1
\end{array}\right)(-\alpha)^{n-1} \\
0 & 0 & 1 & -\left(\begin{array}{l}
3 \\
2
\end{array}\right) \alpha & \cdots & \cdots \cdots & \left(\begin{array}{c}
n \\
2
\end{array}\right)(-\alpha)^{n-2} \\
0 & 0 & 0 & 1 & \cdots & \cdots \cdots & \left(\begin{array}{c}
n \\
3
\end{array}\right)(-\alpha)^{n-3} \\
\vdots & & \ddots & & & & \\
0 & 0 & 0 & 0 & 1 & -\left(\begin{array}{c}
p+1 \\
p
\end{array}\right) \alpha \cdots & \left(\begin{array}{c}
n \\
p
\end{array}\right)(-\alpha)^{n-p} \\
\vdots & & \ddots & & & & \\
0 & 0 & 0 & 0 & 0 & \cdots \cdots \cdots & -\left(\begin{array}{c}
n \\
n-1
\end{array}\right) \alpha \\
0 & 0 & 0 & 0 & 0 & \cdots \cdots \cdots \cdots & 1
\end{array}\right)\left(\begin{array}{c}
a_{0} \\
a_{1} \\
a_{2} \\
a_{3} \\
\vdots \\
a_{p} \\
\vdots \\
a_{n-1} \\
a_{n}
\end{array}\right)=\left(\begin{array}{c}
b_{0} \\
b_{1} \\
b_{2} \\
b_{3} \\
\vdots \\
b_{p} \\
\vdots \\
b_{n-1} \\
b_{n}
\end{array}\right) .
\end{aligned}
$$

In particular, $a_{0}=f(\alpha)$. In the case when $f(y)=\chi_{y}(X)$ is the Hirzebruch $\chi_{y}$-genus, i.e.,

$$
\chi_{y}(X)=\chi^{0}(X)+\chi^{1}(X) y+\chi^{2}(X) y^{2}+\cdots+\chi^{p}(X) y^{p}+\cdots+\chi^{n}(X) y^{n}
$$

(thus each $b_{i}=\chi^{i}(X)$ ), for a compact nonsingular variety or a compact rational homology manifold $X \chi_{-1}(X)=\chi(X)$ and $\chi_{1}(X)=\sigma(X)$ are, respectively, the first constant term $a_{0}, d_{0}$ of the following Taylor expansion of $\chi_{y}(X)$ at $y=-1, y=1$ : 
1. $\chi_{y}(X)=\chi_{-1}(X)+a_{1}(X)(y+1)+a_{2}(X)(y+1)^{2}+\cdots+a_{n}(X)(y+1)^{n}$,

2. $\chi_{y}(X)=\chi_{1}(X)+d_{1}(X)(y-1)+d_{2}(X)(y-1)^{2}+\cdots+d_{n}(X)(y-1)^{n}$.

As to the Taylor expansion of $\chi_{y}(X)$ at $y=-1$, many people have already studied first few terms $a_{1}(X), a_{2}(X), a_{3}(X), a_{4}(X)$ (e.g., see $[\mathbf{1 7}, \mathbf{2 4}, \mathbf{2 8}, \mathbf{1 2}, \mathbf{1 5}, \mathbf{1 6}]$, etc.): for a compact complex manifold $V$ of dimension $n$

1. $a_{1}(V)=-\frac{1}{2} n \chi(V)$,

2. $a_{2}(V)=\frac{1}{12}\left[\frac{1}{2} n(3 n-5) \chi(V)+c_{n-1} c_{1}\right]$,

3. $a_{3}(V)=-\frac{1}{24}\left[\frac{n(n-2)(n-3)}{2} \chi(V)+(n-2) c_{1} c_{n-1}\right]$,

4. $a_{4}(V)=-\frac{1}{5760}\left[n\left(15 n^{3}-150 n^{2}+485 n-502\right) \chi(V)+4\left(15 n^{2}-85 n+108\right) c_{1} c_{n-1}\right.$

$$
\left.+8\left(c_{1}^{2}+3 c_{2}\right) c_{n-2}-8\left(c_{1}^{3}-3 c_{1} c_{2}+3 c_{3}\right) c_{n-3}\right] .
$$

Here we denote the Chern numbers $\left(c_{i_{1}}(V) \cdots c_{i_{j}}(V)\right) \cap[V]$ simply by $c_{i_{1}} \cdots c_{i_{j}}$, where $i_{1}+\cdots i_{j}=n=\operatorname{dim} V$.

Remark 5.2. In [17] Libgober and Wood compute all the coefficients $a_{i}(V)(1 \leqq i \leqq n)$ in the case when $1 \leqq n=\operatorname{dim} V \leqq 6$. We recently found Debarre's paper [7], in which he computes all the coefficients $a_{i}(V)$ for $1 \leqq n \leqq 9$.

It follows from the above formula (1) that obtaining a general formula for the coefficient $\chi^{p}(X)$ is equivalent to obtaining a general formula for the coefficient $a_{p}(X)$, and thus it seems to be quite hard (or almost impossible) to get a general formula for $\chi_{0}(X)=\chi^{0}(X)=\chi^{a}(X)$. Which would suggest that it would be quite hard to get a general explicit formula for $\chi_{y}(X)$ like some cases done in $\S 4$. The merit of considering the Taylor expansion of $\chi_{y}(X)$ at $y=-1$ is that one can compute or express concretely at least first few or several coefficients $a_{i}(X)$ unlike the case of the coefficient $\chi^{i}(X)$. This kind of thing is called "-1-phenomena" in Li's recent works $[15,16]$.

Motivated by the higher Euler-Poincaré characteristic, we will introduce the following:

\section{Definition 5.3.}

$$
\chi_{y}^{(p)}(X):=\frac{1}{p !} \frac{d^{p}}{d y^{p}}\left(\chi_{y}(X)\right)
$$

is called the $p$-th derived Hirzebruch $\chi_{y}$-genus of $X$.

$$
T_{y *}^{(p)}(X):=\frac{1}{p !} \frac{d^{p}}{d y^{p}}\left(T_{y *}(X)\right)
$$

is called the p-th derived motivic Hirzebruch class of $X$.

As to the motivic Hirzebruch class transformation $T_{y *}: K_{0}(\mathcal{V} / X) \rightarrow H_{*}(X) \otimes$ $\mathbb{Q}[y]$, we define the $p$-th derived motivic Hirzebruch class transformation as the fol- 
lowing composition:

$$
T_{y *}^{(p)}:=\frac{d^{p}}{d y^{p}} \circ T_{y *}: K_{0}(\mathcal{V} / X) \stackrel{T_{y *}}{\longrightarrow} H_{*}(X) \otimes \mathbb{Q}[y] \stackrel{\frac{d^{p}}{d y^{p}}}{\longrightarrow} H_{*}(X) \otimes \mathbb{Q}[y] .
$$

The naturality of this transformation is clear because of the naturality of the differential $\mathcal{D}:=\frac{d}{d y}$, i.e., the commutativity of the following diagram for a proper morphism $f: X \rightarrow Y:$

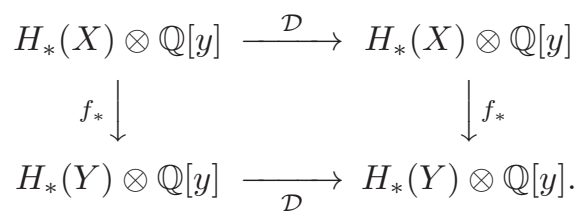

Which implies that for any $p>0$ and $\mathcal{D}^{p}=\overbrace{\mathcal{D} \circ \mathcal{D} \circ \cdots \circ \mathcal{D}}^{p}=\frac{d^{p}}{d y^{p}}$ the following commutes:

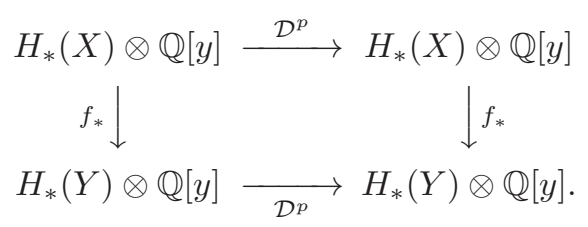

Remark 5.4. We note the following equalities:

1. $\chi_{y}^{(p)}(X)=T_{y *}^{(p)}([X \rightarrow p t])$, where $X$ is compact.

2. $T_{y *}^{(p)}(X)=T_{y *}^{(p)}\left(\left[X \stackrel{\mathrm{id}_{X}}{\longrightarrow} X\right]\right)$.

Remark 5.5. Note that if $X$ is a toric variety, $\chi_{y}^{(p)}(X)$ and $T_{y}^{(p)}(X)$ are explicitly calculated in [21, Theorem 1.1 and Formula $(1.7)]$; e.g., $(-1)^{p} \cdot \chi_{y}^{(p)}(X)$ is just the number of the $p$-dimensional torus orbits.

The following formula follows from the commutativity of the motivic Hirzebruch class and the cross products, and taking derivatives of cross products is similar to taking derivatives of product of two functions:

Theorem 5.6. For two varieties $X, Y$, we have

$$
\left.\left.T_{y^{*}}^{(p)}([V \rightarrow X] \times[W \rightarrow Y])\right)=\sum_{i=0}^{p}\left(\begin{array}{c}
p \\
i
\end{array}\right) T_{y *}^{(i)}([V \rightarrow X]) \times T_{y *}^{(p-i)}([W \rightarrow Y])\right) .
$$

Corollary 5.7. For two varieties the following hold:

1. $\chi_{y}^{(p)}(X \times Y)=\sum_{i=0}^{p}\left(\begin{array}{c}p \\ i\end{array}\right) \chi_{y}^{(i)}(X) \cdot \chi_{y}^{(p-i)}(Y)$, where $X$ and $Y$ are compact.

2. $T_{y *}^{(p)}(X \times Y)=\sum_{i=0}^{p}\left(\begin{array}{c}p \\ i\end{array}\right) T_{y *}^{(i)}(X) \times T_{y *}^{(p-i)}(Y)$.

Remark 5.8. Note that the formula (1) of Corollary 5.7 follows directly from the multiplicativity of $\chi_{y}$. 
$\chi_{y}: K_{0}(\mathcal{V} / X) \rightarrow \mathbb{Q}[y]$ is a group (in fact, ring) homomorphism, thus the EulerPoincaré characteristic $\chi_{-1}=\chi: K_{0}(\mathcal{V} / X) \rightarrow \mathbb{Z}(\subset \mathbb{Q})$ is a homomorphism for complex algebraic varieties. We recall that the Euler-Poincaré characteristic is the alternating sum of the Betti numbers $b_{i}(X)$. In fact, in [23] McCrory and Parusiński proved that for real algebraic varieties the Betti number can be "captured" as a group homomorphism and thus the Poincaré polynomial can be also "captured" as a group homomorphism.

Theorem 5.9 (McCrory and Parusiński). There is a unique group homomorphism

$$
\beta_{i}: K_{0}\left(\mathcal{V}_{\mathbb{R}}\right) \rightarrow \mathbb{Z}
$$

such that for a compact variety $X, \beta_{i}(X)=b_{i}(X)$ is the usual Betti number.

$$
\mathcal{P}(-)(t):=\sum_{i} \beta_{i} t^{i}: K_{0}\left(\mathcal{V}_{\mathbb{R}}\right) \rightarrow \mathbb{Z}[t]
$$

is a unique group homomorphism such that for a compact variety $X$

$$
\mathcal{P}(X)(t)=\sum_{i} \beta_{i}(X) t^{i}=\sum_{i} b_{i}(X) t^{i}=P_{X}(t)
$$

is the usual Poincaré polynomial.

Using this theorem, we can see that

$$
\chi_{(p)}(-):=\left.\frac{d^{p}}{d t^{p}}(\mathcal{P}(-)(t))\right|_{-1}: K_{0}\left(\mathcal{V}_{\mathbb{R}}\right) \rightarrow \mathbb{Z}
$$

is a homomorphism version of Ramachandran's $p$-th higher Euler characteristic.

At the moment a natural transformation ?: $K_{0}\left(\mathcal{V}_{\mathbb{R}} / X\right) \rightarrow \mathbb{H}_{*}(X) \otimes \mathbb{Z}[t]$ has not been constructed or found yet, thus we do not have a natural transformation version of Ramachandran's $p$-th higher Euler characteristic either, and the above $p$-th derived motivic Hirzebruch class transformation is the only one which is available.

\section{Acknowledgments}

We would like to thank the referee for many valuable comments and suggestions.

\section{References}

[1] P. Aluffi and J.-P. Brasselet, Une nouvelle preuve de la concordance des classes définies par M.H. Schwartz et par R. MacPherson, Bull. Soc. Math. France 136 (2008), 159-166.

[2] P. Baum, W. Fulton and R. MacPherson, Riemann-Roch for singular varieties, Publ. Math. Inst. Hautes Études Sci. 45 (1975), 101-145.

[3] J.-P. Brasselet, J. Schürmann and S. Yokura, Hirzebruch classes and motivic Chern classes for singular spaces, J. Topol. Anal. 2, No. 1 (2010), 1-55.

[4] J.-P. Brasselet, J. Schürmann and S. Yokura, Classes de Hirzebruch et classes de Chern motiviques, C. R. Acad. Sci. Paris, Ser. I 342 (2006), 325-328.

[5] J.-P. Brasselet and M.-H. Schwartz, Sur les classes de Chern d'une ensemble analytique complexe, Astérisque 82-83 (1981), 93-148. 
[6] S.E. Cappell and J.L. Shaneson, Stratifiable maps and topological invariants, J. Amer. Math. Soc. 4 (1991), 521-551.

[7] O. Debarre, Cohomological characterizations of the complex projective space, arXiv:1512.04321.

[8] R. Geoghegan and A. Nicas, Higher Euler characteristics. I, Enseign. Math. 41 (1995), 3-62.

[9] M. Goresky and R. MacPherson, Intersection homology theory, Topology 149 (1980), 155-162.

[10] F. Hirzebruch, Topological Methods in Algebraic Geometry, 3rd ed. (1st German ed. 1956), Springer-Verlag, 1966.

[11] F. Hirzebruch, T. Berger and R. Jung, Manifolds and Modular Forms, Vieweg, 1992.

[12] F. Hirzebruch, On the Euler Characteristic of Manifolds with $c_{1}=0$. A letter to V. Gritsenko, Algebra i Analiz 11 (1999), 126-129.

[13] D. Kotschick, Characteristic numbers of algebraic varieties, Proc. Natl. Acad. Sci. USA 106 (2009), 10114-10115.

[14] D. Kotschick, Topologically invariant Chern numbers of projective varieties, Adv. Math. 229 (2012), 1300-1312.

[15] P. Li, The Hirzebruch $\chi_{y}$-genus and Poincaré polynomial revisited, arXiv: 1409.2911v1.

[16] P. Li, -1-Phenomena for the pluri $\chi_{y}$-genus and elliptic genus, Pacific J. Math. 273 (2015), 331-351.

[17] A.S. Libgober and J.W. Wood, Uniqueness of the complex structure on Käbler manifolds of certain homotopy types, J. Differential Geom. 32 (1990), 139-154.

[18] E. Looijenga, Motivic measures, "Séminaire Bourbaki", Astérisque 276 (2002), 267-297.

[19] R. MacPherson, Chern classes for singular algebraic varieties, Ann. of Math. 100 (1974), 423-432.

[20] R. MacPherson, Characteristic classes for singular varieties, "Proceedings of the 9-th Brazilian Mathematical Colloquium" (Poços de Caldas 1973), Vol. II, Instituto de Matemática Pura e Aplicada, São Paulo (1977), 321-327.

[21] L. Maxim and J. Schürmann, Characteristic classes of singular toric varieties, Comm. Pure Appl. Math. 68 (2015), 2177-2236.

[22] L. Maxim, M. Saito and J. Schürmann, Symmetric products of mixed Hodge modules, J. Math. Pures Appl. 96 (2011), 462-483.

[23] C. McCrory and A. Parusiński, Virtual Betti numbers of real algebraic varieties, C. R. Acad. Sci. Paris, Ser. I 336 (2003), 763-768.

[24] M.S. Narasimhan and S. Ramanan, Generalized Prym varieties as fixed points, J. Indian Math. Soc. (N.S.) 39 (1975), 1-19.

[25] C. Peters, Motivic Aspects of Hodge Theory, TIFR - Lecture Notes on Math. 92, Narosa Publ. Company \& AMS (2010), 150 pp.

[26] N. Ramachandran, Higer Euler characteristics: Variations on a theme of Euler, Homology Homotopy Appl. 18 No. 1, (2016), 231-246. 
[27] M. Saito, Mixed Hodge modules, Publ. RIMS, Kyoto Univ. 26 (1990), 221-333.

[28] S. Salamon, On the cohomology of Kähler and hyper-Kähler manifolds, Topology 35 (1996), 137-155.

[29] J. Schürmann, Characteristic classes of mixed Hodge modules, "Topology of Stratified Spaces", MSRI Publ. 58, Cambridge Univ. Press (2011), 419-470.

[30] J. Schürmann and S. Yokura, A survey of characteristic classes of singular spaces, "Singularity Theory" (D. Cheniot et al., ed.), dedicated to Jean-Paul Brasselet on his 60th birthday, Proceedings of the 2005 Marseille Singularity School and Conference, World Scientific (2007), 865-952.

[31] M.-H. Schwartz, Classes caractéristiques définies par une stratification d'une variétés analytique complexe, C. R. Acad. Sci. Paris 260 (1965), 3262-3264, 3535-3537.

[32] M.-H. Schwartz, Classes et caractères de Chern des espaces linéaires, Pub. Int. Univ. Lille, 2 Fasc. 3 (1980) and C. R. Acad. Sci. Paris, Sér. I 295 (1982), 399-402.

[33] S. Yokura, On Cappell-Shaneson's homology L-class of singular algebraic varieties, Trans. Amer. Math. Soc. 347 (1995), 1005-1012.

[34] S. Yokura, Motivic characteristic classes, "Topology of Stratified Spaces", MSRI Publ. 58, Cambridge Univ. Press (2011), 375-418.

Jean-Paul Brasselet jean-paul.brasselet@univ-amu.fr

Institute de Mathématiques de Marseille, UMR 7373, Campus de Luminy - Case 907, 13288 Marseille Cedex 9, France

Jörg Schürmann jschuerm@math.uni-muenster.de

Westfälische Wilhelms-Universität, Mathematische Institut, Einsteinstrasse 62, 48149 Münster, Germany

Shoji Yokura yokura@sci.kagoshima-u.ac.jp

Department of Mathematics and Computer Science, The Graduate School of Science and Engineering, Kagoshima University, 1-21-35 Korimoto, Kagoshima 890-0065, Japan 